\title{
An Improved GA for solving multiple depot VRP
}

\author{
Yudong ZHANG, ${ }^{1 *}$ Genlin $\mathrm{JI},{ }^{1}$ Shuihua WANG, ${ }^{12}$ Preetha PHILLIPS, ${ }^{3}$ William \\ WANG $^{4}$, Elizabeth LEE ${ }^{5}$ \\ ${ }^{1}$ School of Computer Science and Technology, Nanjing Normal University, Nanjing, Jiangsu \\ 210023, China \\ ${ }^{2}$ School of Electronic Science and Engineering, Nanjing University, Nanjing, Jiangsu 210046, \\ China \\ ${ }^{3}$ School of Natural Sciences and Mathematics, Shepherd University, Shepherdstown, WV 25443, \\ USA \\ ${ }^{4}$ Waynesburg University, Waynesburg, PA 15370, USA \\ ${ }^{5}$ Department of Engineering Technology, Chattanooga State Community College, Chattanooga, \\ TN 37406, USA \\ Email: zhangyudong@njnu.edu.cn
}

Keyword: Vehicle routing problem; genetic algorithm; fitness-scaling; local search

Abstract.Multi-depot vehicle routing problem is a NP-hard combinatorial optimization problem. In this paper, we proposed an improved genetic algorithm (GA), which combined GA with fitnessscaling and local search. The experiments compared the proposed approach with standard GA, simulated annealing, Tabu search, and particle swarm optimization. The results showed that the proposed method was superior to GA, SA, TS, and PSO, w.r.t. solution accuracy.

\section{Introduction}

The Vehicle Routing Problem (VRP) is a whole class of problems that optimize the cost of product delivery by vehicles from depots to customers under certain constraints [1, 2]. In this study, we focus on the Multiple Depot VRP (MDVRP).

The VRP falls within the NP-hard problem. At present, there are two schools of methods. One is the integer programming based methods, which can obtain global optimal solutions with exponentially increasing computation time. Therefore, those methods are infeasible. The other is heuristics-based methods, approximate solutions are found fast enough and sufficiently accurate. Recent reports show that Genetic Algorithm (GA) [3], Simulated Annealing (SA) [4], Tabu search (TS) [5], Particle Swarm Optimization (PSO) [6] can be used to solve VRP. However, the performance of pure SA is not satisfactory [7], the performance of pure TS is generally neither satisfactory nor competitive [8-10]. The discrete version of PSO performs far behind the basic ant colony algorithm $[11,12]$.

In this paper, we proposed an improved GA (IGA) method that combines the advantages of the fitness-scaling mechanism and 2-opt local search movement. The paper aims at improving the solution accuracy.

\section{Problem Definition}

Suppose $S$ represents the number of depots, and $\mathbb{D}$ the index set of depots index

$$
\mathbb{D}=\{1,2, \ldots, S\}
$$

and suppose $U$ is the number of customers, and $\mathbb{C}$ the index set of customers

$$
\mathbb{C}=\{1,2, \ldots, U\}
$$

The node index set $\mathbb{V}$ is the combination of the sets of depots and customers

$$
\mathbb{V}=\mathbb{C} \cup \mathbb{D}=\{1,2, \ldots, S+U\}
$$

where a node is either a depot or a customer. 
Each depot stores and supplies various products. Suppose $T$ denotes the total number of types of products, and $\mathbb{P}$ the product index set

$$
\mathbb{P}=\{1,2, \ldots, T\}
$$

Suppose $Q_{i}$ is the total number of vehicles stationed in depot $i$, we can define $\mathbb{F}_{i}$ the index set of vehicles stationed in depot $i$

$$
\mathbb{F}_{i}=\left\{1,2, \ldots, Q_{i}\right\}
$$

The mathematical formula of MDVRP is written as

$$
f=\sum_{i \in \mathbb{D}} \sum_{k \in \mathbb{F}_{i}} \sum_{m \in \mathbb{V}} \sum_{n \in \mathbb{V}} c_{m n} x_{i k m n}
$$

where $x_{i k m n}$ is a binary variable with 1 representing vehicle $k$ serving depot $i$ visits node $n$ after node $m$, and 0 otherwise. $c_{m n}$ is the distance between node $m$ and node $n$. In addition, MDVRP is subject to following 6 criteria:

$$
\sum_{k \in \mathbb{F}_{i}} \sum_{m \in \mathbb{C}}\left(d_{j m} \sum_{n \in \mathbb{V}} x_{i k m n}\right) \leq s_{i j}, \forall i \in \mathbb{D}, \forall j \in \mathbb{P}
$$

where $d_{j m}$ is the demand of product $j$ for node $m$, and $s_{i j}$ is the supply of product $j$ from depot $i$. The supply of each type of product from the depot should not be less than the corresponding demand from customers served by all vehicles in the same depot.

$$
\sum_{j \in \mathbb{P}} \sum_{m \in \mathbb{C}}\left(d_{j m} v_{j} \sum_{n \in \mathbb{V}} x_{i k m n}\right) \leq e_{i k}, \forall i \in \mathbb{D}, \forall k \in \mathbb{F}_{i}
$$

where $e_{i k}$ denotes the capacity of vehicle $k$ serving depot $i$. The total volume of different products requested by customers served by the same vehicle should not be more than the capacity of the same vehicle.

$$
\sum_{i \in \mathbb{D}} \sum_{k \in \mathbb{F}_{i}} \sum_{m \in \mathbb{V}} x_{i k m n}=1, \forall n \in \mathbb{C}
$$

which requires each customer should be served once and only one vehicle.

$$
\sum_{n \in \mathbb{V}} x_{i k m m}=\sum_{n \in \mathbb{V}} x_{i k m n}, \forall i \in \mathbb{D}, \forall k \in \mathbb{F}_{i}, \forall m \in \mathbb{V}
$$

which requires that any vehicle entered a customer should leave from the same customer.

$$
\sum_{m \in \mathbb{V}} \sum_{n \in \mathbb{V}} x_{i k m n} \leq\left|\mathbb{E}_{k}\right|-1, \forall \mathbb{E}_{k} \subseteq \mathbb{C},\left|\mathbb{E}_{k}\right| \geq 2, \forall i \in \mathbb{D}, \forall k \in \mathbb{F}_{i}
$$

where $\mathbb{E}_{k}$ denotes the set of customers visited by vehicle $k$.

$$
x_{i k m n} \in\{0,1\}, \forall i \in \mathbb{D}, \forall k \in \mathbb{F}_{i}, \forall m \in \mathbb{V}, \forall n \in \mathbb{V}
$$

which requires the value of the decision variable should be binary.

\section{Methodology}

Fitness scaling converts the raw fitness values to a range suitable for better selection. Power-rank fitness scaling strategy $[13,14]$ is selected. Afterwards, 2-opt is an intra-route movement to improve the solutions. It consists of replacing two non-adjacent arcs belonging to the route by two other arcs not belonging to it, by which the connectivity of the route is re-established [15].

Based on aforementioned description, we proposed the IGA, which combined standard GA with fitness-scaling and local search.

\section{Experiments and Results}

The Cordeau dataset contains 33 benchmark problems. We compared the proposed IGA with standard GA, SA, TS, and PSO. For each problem, each algorithm was conducted 50 times. The best results among 50 runs were recorded. The parameters are obtained by trial-and-error method. The results are evaluated by the best (minimum) objective value $f_{\text {best }}$, and the percentage deviation $\left(P_{D}\right)$ defined as

$$
P_{D}=\frac{f_{\text {best }}-f_{K B}}{f_{K B}} \times 100 \%
$$


where $f_{K B}$ represents the known-best objective value, which can be downloaded from the homepage of the Cordeau dataset. Table 1 reports the best (minimum) results and its deviation in terms of percentage by all algorithms for all benchmark problems.

Table 1 Algorithm comparison among 50 runs

\begin{tabular}{lccccc}
\hline Instance & GA & SA & TS & PSO & IGA \\
\hline p01 & 0.0000 & 0.0000 & 0.0093 & 0.0000 & 0.0000 \\
p02 & 0.0000 & 0.0000 & 0.0000 & 0.0000 & 0.0000 \\
p03 & 0.0000 & 0.0000 & 0.0264 & 0.0000 & 0.0000 \\
p04 & 0.0000 & 0.0000 & 0.0000 & 0.0000 & 0.0000 \\
p05 & 0.0000 & 0.0000 & 0.0000 & 0.0000 & 0.0000 \\
p06 & 0.0000 & 0.0000 & 0.0000 & 0.0000 & 0.0000 \\
p07 & 0.0000 & 0.0000 & 0.0000 & 0.0000 & 0.0000 \\
p08 & 0.0000 & 0.0000 & 0.0000 & 0.0000 & 0.0000 \\
p09 & 0.0136 & 0.0000 & 0.0202 & 0.0000 & 0.0000 \\
p10 & 0.0000 & 0.0000 & 0.0000 & 0.0265 & 0.0000 \\
p11 & 0.0600 & 0.0000 & 0.0000 & 0.0290 & 0.0000 \\
p12 & 0.0000 & 0.0000 & 0.0000 & 0.0000 & 0.0000 \\
p13 & 0.0000 & 0.0000 & 0.0000 & 0.0000 & 0.0000 \\
p14 & 0.0000 & 0.0000 & 0.0000 & 0.0000 & 0.0000 \\
p15 & 0.0102 & 0.0127 & 0.0000 & 0.0000 & 0.0000 \\
p16 & 0.0124 & 0.0000 & 0.0315 & 0.0127 & 0.0050 \\
p17 & 0.0098 & 0.0265 & 0.0282 & 0.0176 & 0.0000 \\
p18 & 0.0000 & 0.0292 & 0.0100 & 0.0229 & 0.0000 \\
p19 & 0.0491 & 0.0000 & 0.0000 & 0.0000 & 0.0401 \\
\hline p20 & 0.0356 & 0.0073 & 0.0000 & 0.0433 & 0.0273 \\
p21 & 0.0271 & 0.0211 & 0.1493 & 0.0293 & 0.0227 \\
p22 & 0.0698 & 0.0651 & 0.0686 & 0.0483 & 0.1034 \\
p23 & 0.0045 & 0.1136 & 0.0208 & 0.1363 & 0.0142 \\
pr01 & 0.0020 & 0.0000 & 0.0000 & 0.0000 & 0.0000 \\
pr02 & 0.0000 & 0.0000 & 0.0000 & 0.0000 & 0.0000 \\
pr03 & 0.0000 & 0.0000 & 0.0000 & 0.0000 & 0.0000 \\
pr04 & 0.0000 & 0.0000 & 0.0000 & 0.0000 & 0.0000 \\
\hline pr05 & 0.0000 & 0.0220 & 0.0410 & 0.0303 & 0.0000 \\
pr06 & 0.0503 & 0.0413 & 0.0000 & 0.0000 & 0.0283 \\
pr07 & 0.0000 & 0.0152 & 0.0165 & 0.0000 & 0.0071 \\
pr08 & 0.0593 & 0.0357 & 0.0000 & 0.0000 & 0.0000 \\
pr09 & 0.0000 & 0.0450 & 0.0000 & 0.0311 & 0.0000 \\
pr10 & 0.0000 & 0.0000 & 0.0698 & 0.0554 & 0.0219 \\
\hline & & & & &
\end{tabular}

Results indicated that the proposed IGA method performed the best. It reached the known-best solutions in 24 instances and failed in only 9 instances. In addition, standard GA performed the worst and it won only 20 instances and failed in 13 instances. SA, TS, and PSO won 21 instances and failed in 12 instances.

\section{Conclusions}

In this paper, we proposed an improved GA algorithm in order to solve MDVRP. Thirty-three benchmark problems were used to test the performance of the proposed algorithm. The results showed the superiority of the proposed IGA algorithm to other methods.

\section{Reference}

[1] Y.D. Zhang, L.N. Wu, G. Wei, S.H. Wang, A novel algorithm for all pairs shortest path problem based on matrix multiplication and pulse coupled neural network, Digital Signal Processing, 21 (2011) 517-521.

[2] C. Ping, Q. Youli, H. Houkuan, D. Xingye, A New Hybrid Iterated Local Search for the Open Vehicle Routing Problem, in: Computational Intelligence and Industrial Application, 2008. PACIIA 
'08. Pacific-Asia Workshop on, 2008, pp. 891-895.

[3] Y.D. Zhang, S.H. Wang, G.L. Ji, A Rule-Based Model for Bankruptcy Prediction Based on an Improved Genetic Ant Colony Algorithm, Mathematical Problems in Engineering, (2013) 10.

[4] P. Javier Herrera, G. Pajares, M. Guijarro, J.J. Ruz, J.M. de la Cruz, Combining Support Vector Machines and simulated annealing for stereovision matching with fish eye lenses in forest environments, Expert Systems with Applications, 38 (2011) 8622-8631.

[5] P.K. Nguyen, T.G. Crainic, M. Toulouse, A tabu search for Time-dependent Multi-zone Multitrip Vehicle Routing Problem with Time Windows, European Journal of Operational Research, 231 (2013) 43-56.

[6] Y. Zhang, S. Wang, P. Phillips, G. Ji, Binary PSO with mutation operator for feature selection using decision tree applied to spam detection, Knowledge-Based Systems, 64 (2014) 22-31.

[7] S.W. Lin, K.C. Ying, Z.J. Lee, H.S. Chen, Vehicle Routing Problems with Time Windows Using Simulated Annealing, in: Systems, Man and Cybernetics, 2006. SMC '06. IEEE International Conference on, 2006, pp. 645-650.

[8] J. Tongpang, P. Tantatsanawong, An Application of Tabu Search Algorithms and Genetic Algorithms in Collaborative Logistics Optimization, in: SRII Global Conference (SRII), 2011 Annual, 2011, pp. 699-706.

[9] X.-c. Fan, N.-l. Li, B.-y. Zhang, Z.-h. Liu, Research on vehicle routing problem with soft time windows based on tabu search algorithm, in: Industrial Engineering and Engineering Management (IE\&EM), 2011 IEEE 18Th International Conference on, 2011, pp. 420-423.

[10] J. Ge, T. Wang, H. Wang, The TS\&SS algorithm for vehicle routing problem, in: Control and Decision Conference (CCDC), 2013 25th Chinese, 2013, pp. 396-400.

[11] X. Tan, Y. Ren, Study on the Emergency Rescue VRP Based on Ant Colony Optimization and Generalized Distance, in: Intelligent Systems (GCIS), 2013 Fourth Global Congress on, 2013, pp. 53-57.

[12] J. Tang, J. Guan, Y. Yu, J. Chen, Beam Search Combined With MAX-MIN Ant Systems and Benchmarking Data Tests for Weighted Vehicle Routing Problem, Automation Science and Engineering, IEEE Transactions on, PP (2014) 1-13.

[13] Y. Zhang, L. Wu, S. Wang, UCAV Path Planning by Fitness-Scaling Adaptive Chaotic Particle Swarm Optimization, Mathematical Problems in Engineering, 2013 (2013) 9.

[14] Y. Zhang, L. Wu, S. Wang, UCAV path planning based on FSCABC, Information-An International Interdisciplinary Journal, 14 (2011) 687-692.

[15] F. Alfredo Tang Montané, R.D. Galvão, A tabu search algorithm for the vehicle routing problem with simultaneous pick-up and delivery service, Computers \& Operations Research, 33 (2006) 595-619. 